\title{
Shuttling of Dorsal by Cactus: mechanism and implications
}

Allison E. Schloop ${ }^{1}$, Sophia Carrell-Noel ${ }^{2}$, and Gregory T. Reeves ${ }^{1,2, \ddagger}$

1 - Genetics Program, North Carolina State University, Raleigh, NC

2 - Department of Chemical and Biomolecular Engineering, North Carolina State University, Raleigh, NC

キ - To whom correspondence should be addressed, gtreeves@ncsu.edu

Word count: 4353 


\begin{abstract}
In a developing animal, morphogen gradients act to pattern tissues into distinct domains of cell types. However, despite their prevalence in development, morphogen gradient formation is a matter of debate. In our recent publication, we showed that the Dorsal/NF- $\kappa \mathrm{B}$ morphogen gradient, which patterns the DV axis of the early Drosophila embryo, is partially established by a mechanism of facilitated diffusion. This mechanism, also known as "shuttling," occurs when a binding partner of the morphogen facilitates the diffusion of the morphogen, allowing it to accumulate at a given site. In this case, the inhibitor Cactus/IкB facilitates the diffusion of Dorsal/NF- $\mathrm{kB}$. In the fly embryo, we used computation and experiment to not only show that shuttling occurs in the embryo, but also that it enables the viability of embryos that inherit only one copy of dorsal maternally. Here we further discuss our evidence behind the shuttling mechanism, the previous literature data explained by the mechanism, and how it may also be critical for robustness of development. Finally, we describe an interaction between Dorsal and BMP signaling that is likely affected by shuttling.
\end{abstract}

Keywords: Dorsal, Cactus, Toll, shuttling, diffusion, embryo, morphogen, dorsal-ventral axis, development

\title{
Introduction
}

Animal development is orchestrated by morphogen gradients which help to pattern tissues based on location and concentration. The formation of these gradients has been a subject of intense scrutiny [1-3]. In Drosophila, Dorsal (Dl), a maternally provided transcription factor, acts as a morphogen to regulate patterning of the dorsal-ventral (DV) axis of the early (1-3 hr 
old) embryo [4]. During these stages of embryogenesis, the nuclei rapidly divide without cytokinesis. These nuclei share a common cytoplasm, and thus, intracellular proteins may diffuse from one nucleus to another. The first nine nuclear division cycles after fertilization occur within the volume of the embryo. After nuclear cycle (nc) 9, the nuclei migrate to the periphery of the embryo, forming a syncytial blastoderm. Four further nuclear divisions occur, and during nc 14, cell membranes introgress between the nuclei in a process called cellularization. Roughly 45 minutes after the start of nc 14, gastrulation takes place, after which, Dl signaling ceases.

While initially uniformly distributed, a nuclear concentration gradient of Dl begins to develop during nuclear cycle (nc) 10. This ultimately results in high concentrations in ventral nuclei and a spatial gradient that decreases to basal concentrations in dorsal nuclei [4-9]. Establishment of this gradient leads to the activation of different genes around the DV axis depending on the concentration of D1 $[10,11]$. While in the cytoplasm, Dl is bound to Cactus (Cact), which prevents movement into the nucleus; degradation of Cact through Toll signaling on the ventral side releases Dl from Cact and allows it to enter [10,12-15]. Taken together, this information suggests that a depletion of $\mathrm{Dl}$ in the cytoplasm around the ventral-most nuclei would occur, creating a cytoplasmic Dl gradient opposite to that of nuclear Dl $[4,16]$. However, recent work indicates that there is an accumulation of total Dl (nuclear Dl+cytoplasmic Dl) on the ventral side of the embryo as development progresses (Fig. 1) [5,17]. The current paradigm -Toll signaling, the degradation of Cact, and the local nuclear import of Dl -- cannot explain this accumulation. Therefore, through both computational and experimental means, we have suggested that a facilitated diffusion, or shuttling, mechanism is responsible for the accumulation of total Dl with Cact acting as a carrier molecule [17]. In such a mechanism, a carrier molecule, 
in this case Cact, facilitates the diffusion of the primary molecule (D1) against its own concentration gradient.

\section{[Figure 1 near here]}

Shuttling mechanisms have already been described in conjunction with the formation of signaling gradients in the Drosophila embryo; two examples include gradient formation in the Dpp/BMP signaling pathway and establishment of the Spätzle (Spz) gradient. In the Dpp/BMP signaling pathway, the shuttling mechanism, in which an inhibitory complex of Short gastrulation (Sog) and Twisted gastrulation (Tsg) facilitates transport of the BMP ligand Dpp to the dorsal region of the embryo, is well established [18-25]. Dpp signaling begins early in nc 14 as a weak, broad distribution on the dorsal half of the embryo, but becomes highly intense and concentrated at the dorsal midline by late nc 14 [24]. Laterally expressed Sog and Tsg form a complex with Dpp and a second BMP ligand, Scw. The complex prevents Dpp signalling, prohibits movement away from the dorsal region, and protects Dpp from degradation. Loss of either Sog or Tsg results in a failure to accumulate Dpp signaling along the dorsal midline [20]. Tolloid, a metalloprotease that cleaves Sog to release active BMP ligand, is expressed on the dorsal half of the embryo, and is required for shuttling [21].

For Spz, the Toll ligand, a theoretical self-organized shuttling mechanism has been proposed [16] in which Spz is cleaved into two parts that rejoin to form a complex. This complex can experience several outcomes, one of which is dissociation into an inhibitory molecule $(\mathrm{N}$ Spz) and an active molecule (C-Spz). Through modeling work, Haskel-Ittah et al. have suggested that these two molecules can reassociate to form an inactive complex. This complex freely diffuses around the DV axis, but upon reaching the ventral region of the embryo, the N-Spz of 
this inactive complex is degraded. This mechanism would cause an accumulation of the active molecule, C-Spz, on the ventral side.

The shuttling mechanism is not limited to Drosophila. In Xenopus and Danio, the Sog homolog Chordin performs a similar role in concentrating BMP activity to the ventral side of these embryos [26]. This evidence indicates that not only is shuttling important for the establishment of multiple morphogen gradients, it also occurs during gradient establishment in many organisms.

\section{Methods}

Fly lines

For maternal over expression of the Mad and Medea constructs, we used a mat $\alpha-\mathrm{Gal} 4$ line available from the Drosophila Bloomington Stock Center $\left(\mathrm{w}\left[{ }^{\star}\right] ; \mathrm{P}\{\mathrm{w}[+\mathrm{mC}]=\right.$ matalpha4-GAL4-VP16 \}V37, BS\#7063). We are grateful to Laurel Raftery for kindly providing the UAS-Med and UAS-Mad lines [27].

\section{Fluorescent antibody staining}

Standard protocols were followed for fixation of 2-4 hr embryos in $37 \%$ formaldehyde solution [28].Fluorescent antibody staining was performed according to standard protocols [28], with primary antibodies against Dl (1:10 dilution; DSHB anti-dorsal 7A4) and histone (1:5000 dilution; abcam ab1791). The anti-Dl monoclonal antibody (DSHB Hybridoma Product antidorsal 7A4) was deposited to the DSHB by R. Steward [15], and was obtained from the Developmental Studies Hybridoma Bank, created by the NICHD of the NIH and maintained at The University of Iowa, Department of Biology, Iowa City, IA 52242. Secondary antibodies 
used were donkey anti-mouse-488 (1:500 dilution; Invitrogen A21202) and donkey anti-rabbit546 (1:500 dilution; Invitrogen A10040),

\section{Mounting and imaging embryos}

Previously-published protocols for mounting and imaging embryos were used [29]. Briefly, fixed embryos were manually cross sectioned in $70 \%$ glycerol using a razor blade. The anterior and posterior $1 / 3$ rds of the embryo were removed, and the remaining $1 / 3$ (trunk portion of the embryo) was mounted vertically on a microscope slide. Two pieces of double-sided tape were used on either side of the embryos to create a vertical space so that the coverslip would not deform the embryos. Embryos were imaged using a Zeiss 710 laser scanning confocal microscope. 10-20 z-slices were taken for each embryo.

\section{Image analysis}

Image analysis was performed according to our previously published protocols [30]. Briefly, the nuclei were segmented, and the Dl intensity in the nuclei was measured. Gradient width was determined by fitting the $\mathrm{Dl}$ gradient to a Gaussian-like shape $[5,6,30]$. For figures $2 \mathrm{C}$ and 3A,B, each z-slice was normalized independently to accurately depict the shape of the gradient.

\section{Evidence of shuttling in the Dorsal gradient}

The D1/Cact system follows the four features necessary for a shuttling mechanism to occur (see Fig. 2A) [17,31]: (1) Dl, the primary molecule, binds to Cact, a carrier molecule, 
preventing its capture or degradation; (2) the complex of Dl/Cact can diffuse on a global scale;

(3) the complex is degraded in a spatially-dependent manner, which for D1/Cact is through Toll signalling on the ventral side; and (4) once free of its carrier, the primary molecule can be captured (in this case, by the nucleus) or degraded. The fourth feature prevents free cytoplasmic Dl from diffusing back to the dorsal side (counter-diffusion). Any system with these four features, balanced in the proper way, may exhibit shuttling. Indeed, previous models of the Dl/Cact system, which captured these four features, exhibited shuttling without the express intention of the modelers [32].

[Figure 2 near here]

Each of these features is well established in the Dl/Cact system -- including point (2), which we confirmed by live imaging of photoactivatable GFP-tagged Dl; [17] -- yet it remained to be seen whether these four processes were balanced in such a way that shuttling occurred in an appreciable manner. Our model of the Dl/Cact system suggested that, if shuttling did occur appreciably, then decreasing the ventrally-directed flux due to shuttling would result in one of a hallmark set of phenotypes, depending on the degree to which the flux lowered [17]. By slowing the diffusion of $\mathrm{Dl}$ and Dl/Cact complex, the Dl gradient would widen, become flat on top, or (most severely) split into two peaks (Fig. 2B). In a typical morphogen gradient system (without shuttling), in which the morphogen is produced locally and spreads, lowering the diffusion is expected to narrow the gradient. In contrast, in a shuttling system, diffusion occurs against the gradient of the morphogen, and thus, lowering diffusion would widen the gradient and prevent proper peak accumulation. In a series of experiments in which Dl was tagged with bulky molecules, we found a correlation between widening of the Dl gradient and the degree to which 
the bulky tags were expected to lower Dl/Cact diffusion [17]. While widening of the gradient is the weakest of the predicted hallmark phenotypes of shuttling, the correlation was telling.

In addition to reduced diffusivity resulting in a hallmark shuttling phenotypes, it was also shown that embryos from heterozygous $d l$ null mothers exhibited the hallmark phenotypes to varying degrees (Fig. 2C) [6,17,32]. However, no previous model of the Dl gradient, including our initial models, could reproduce this phenotype when lowering only the dosage of D1 [17,3234]. The Dl gradients from each of the models were predicted to globally scale with dosage (a half dose of maternal $d l$ resulted in a $50 \%$ reduction of the gradient, everywhere), so that changes in shape, including widening or flattening, were never observed. On the other hand, we discovered that if the concentration of active Toll receptors were sufficiently low such that they could be saturated by Dl/Cact complexes, the change in gradient shape could possibly be explained [17]. In wildtype embryos, saturation in lateral regions allows accumulation of Dl at the ventral midline and normal gradient formation (Fig. 2D). However, when maternal $d l$ production is reduced ( $d l$ heterozygous embryos), unsaturated Toll receptors trap what limited D1/Cact complexes are available, preventing accumulation and normal gradient formation (Fig. $2 \mathrm{E})$. The altered $\mathrm{Dl}$ gradient shape in $d l$ heterozygous embryos is partially restored to the Gaussian-like shape when $T l$ levels are reduced [17].

Hallmark shuttling phenotypes have also been previously observed in several other, seemingly unrelated instances. These include when the domain of active Toll receptors is widened and when the Dl gradient is ectopically expressed along the anterior-posterior axis [17]. In each of these cases, the combination of shuttling and Toll saturation is adequate to explain these observations. 
Beyond the hallmark phenotypes observed when the Dl gradient is measured, the shuttling mechanism also allows for intuition regarding a litany of literature observations that were previously unexplained. For example, $d l$ transgene fusions to $l a c Z$ are antimorphic, which is readily explained by the D1- $\beta$ galactosidase forming bukly groups by self-association $[17,35]$. As another example, a greatly expanded domain of active Toll results in the duplication of the ventral furrow at gastrulation [36]; this arises as an extreme instance of the split peak phenotype [17].

\section{Importance of shuttling}

The evidence for shuttling and Toll saturation strongly suggest the four features necessary for shuttling are indeed balanced such that Dl shuttling occurs appreciably in the embryo. On the other hand, given that asymmetric Toll signaling alone, without shuttling, is sufficient to explain the development of a strong ventral-to-dorsal bias in the Dl nuclear concentration, it raises the question of whether a shuttling mechanism is required for proper gene expression in the wildtype embryo. If so, then it stands to reason that cact function would be necessary to accumulate $\mathrm{Dl}$ to the peak levels required for the expression of the ventral-most genes, such as sna and twi. However, seminal work studying Cact function found that the $t w i$ domain is expanded in embryos from cact null mothers [37]. On the other hand, recent quantitative measurements of the $\mathrm{Dl}$ gradient in embryos from mothers with severe loss of cact function are consistent with shuttling being required for proper sna expression [38]. Further quantitative study of the precise distribution of $\mathrm{Dl}$ in cact mutants is required to answer this question.

Another question that may arise is whether shuttling is important in other cases, such as to make the Dorsal gradient more robust. Our findings suggest that shuttling is indeed required to 
accumulate peak levels of Dl in embryos from mothers heterozygous for $d l$. In embryos with compromised shuttling, in the background of maternally heterozygous $d l$, the $\mathrm{Dl}$ gradient is severely perturbed in shape and width. Furthermore, the sna expression domain is either absent or narrowed to the point of loss of embryonic viability [17].

In addition, one of the hallmark phenotypes of shuttling, in which the Dl gradient splits into two peaks, has been found in the context of other perturbations. This may imply that shuttling provides a role in those processes. For example, when investigating the scaling properties of the Dl gradient, a split-peak of the Dl nuclear gradient was found in the largest embryos (Fig. 3A) [39]. Also, sna expression boundaries in these embryos did not scale with size. One possibility is that shuttling allows for size-dependent scaling of the Dl gradient within certain ranges; if the embryo size exceeds certain limits, the shuttling-dependent scaling mechanism fails.

[Figure 3 near here]

Furthermore, we have found that maternally over-expressing components of the Dpp pathway results in hallmark shuttling phenotypes. We used a maternal Gal4 driver to overexpress the BMP signal transduction components Mad or Medea (see Methods); the resulting embryos had widened Dl gradients, while some had flat tops or double peaks (Fig. 3B,C). Thus, it is possible that the shuttling mechanism also somehow mediates the interactions of the maternal Dpp pathway with the Dl gradient [38,40-43].

\section{Conclusions}

The shuttling phenomenon carries with it many counter-intuitive observations, such as a protein classically construed as an inhibitor that can also potentiate the highest levels of signaling 
[19-21,23,24,38]. Even so, the phenomenon of shuttling arises naturally when only a handful of sufficient conditions are met: an inhibitor must bind, the complex must be able to diffuse, there must be a sink for the complex, and the shuttled molecule must not participate in significant counter-diffusion. Each of these processes are known to operate in the early Drosophila embryo for the Dl/Cact system, and are balanced in such a way that shuttling of Dl by Cact occurs appreciably.

The shuttling mechanism for the Dl gradient appears to be a modest revision of our understanding of Dl gradient formation; however, the mechanism is impactful in many ways. It explains how the Dl gradient levels in the ventral-most nuclei continually grow over time, as well as a litany of other previously unexplained literature observations. Furthermore, it appears to be a crucial mechanism for the robustness of embryo development with respect to several common perturbations, including maternal $d l$ dosage compensation, size-dependent scaling, and interactions with other signaling pathways. Future work on the Dl gradient may uncover still more perturbations with phenotypes consistent with the hallmarks of shuttling. 


\section{Acknowledgements}

We thank Dr. Thomas Jacobsen for initial discussions of the manuscript.

\section{Funding}

During this work, AES was partially supported by the National Institutes of Health [grant R21-HD092830], SC-N was partially supported by the U.S. Department of Education [Graduate Assistance in Areas of National Need Biotechnology Fellowship P200A100004] and the National Science Foundation [grant CBET-1254344]. GTR was partially supported by the National Science Foundation [grant CBET-1254344] and the National Institutes of Health [grant R21-HD092830].

\section{Disclosure of interest}

The authors report no conflict of interest.

\section{References}

[1] Christian JL. Morphogen gradients in development: from form to function. Wiley Interdiscip. Rev. Dev. Biol. 2012;1:3-15.

[2] Müller P, Rogers KW, Yu SR, et al. Morphogen transport. Development. 2013;140:16211638.

[3] Wartlick O, Kicheva A, González-Gaitán M. Morphogen gradient formation. Cold Spring Harb. Perspect. Biol. 2009;1:a001255. 
[4] Roth S, Stein D, Nüsslein-Volhard C. A gradient of nuclear localization of the dorsal protein determines dorsoventral pattern in the Drosophila embryo. Cell. 1989;59:1189-1202.

[5] Reeves GT, Trisnadi N, Truong TV, et al. Dorsal-ventral gene expression in the Drosophila embryo reflects the dynamics and precision of the dorsal nuclear gradient. Dev. Cell. $2012 ; 22: 544-557$.

[6] Liberman LM, Reeves GT, Stathopoulos A. Quantitative imaging of the Dorsal nuclear gradient reveals limitations to threshold-dependent patterning in Drosophila. Proc. Natl. Acad. Sci. U. S. A. 2009;106:22317-22322.

[7] Steward R, Zusman SB, Huang LH, et al. The dorsal protein is distributed in a gradient in early drosophila embryos [Internet]. Cell. 1988. p. 487-495. Available from: http://dx.doi.org/10.1016/0092-8674(88)90035-9.

[8] Steward R. Relocalization of the dorsal protein from the cytoplasm to the nucleus correlates with its function [Internet]. Cell. 1989. p. 1179-1188. Available from: http://dx.doi.org/10.1016/0092-8674(89)90773-3.

[9] Rushlow CA, Han K, Manley JL, et al. The graded distribution of the dorsal morphogen is initiated by selective nuclear transport in Drosophila. Cell. 1989;59:1165-1177.

[10] Reeves GT, Stathopoulos A. Graded dorsal and differential gene regulation in the Drosophila embryo. Cold Spring Harb. Perspect. Biol. 2009;1:a000836.

[11] Stathopoulos A, Levine M. Genomic regulatory networks and animal development. Dev. Cell. 2005;9:449-462. 
[12] Bergmann A, Stein D, Geisler R, et al. A gradient of cytoplasmic Cactus degradation establishes the nuclear localization gradient of the dorsal morphogen in Drosophila. Mech.

Dev. 1996;60:109-123.

[13] Belvin MP, Jin Y, Anderson KV. Cactus protein degradation mediates Drosophila dorsalventral signaling. Genes Dev. 1995;9:783-793.

[14] Reach M, Galindo RL, Towb P, et al. A gradient of cactus protein degradation establishes dorsoventral polarity in the Drosophila embryo. Dev. Biol. 1996;180:353-364.

[15] Whalen AM, Steward R. Dissociation of the dorsal-cactus complex and phosphorylation of the dorsal protein correlate with the nuclear localization of dorsal. J. Cell Biol. $1993 ; 123: 523-534$.

[16] Haskel-Ittah M, Ben-Zvi D, Branski-Arieli M, et al. Self-organized shuttling: generating sharp dorsoventral polarity in the early Drosophila embryo. Cell. 2012;150:1016-1028.

[17] Carrell SN, O’Connell MD, Jacobsen T, et al. A facilitated diffusion mechanism establishes the Dorsal gradient. Development. 2017;144:4450-4461.

[18] Shimmi O, Umulis D, Othmer H, et al. Facilitated transport of a Dpp/Scw heterodimer by Sog/Tsg leads to robust patterning of the Drosophila blastoderm embryo. Cell. $2005 ; 120: 873-886$.

[19] Eldar A, Dorfman R, Weiss D, et al. Robustness of the BMP morphogen gradient in Drosophila embryonic patterning. Nature. 2002;419:304-308.

[20] Holley SA, Neul JL, Attisano L, et al. The Xenopus Dorsalizing Factor noggin 
Ventralizes Drosophila Embryos by Preventing DPP from Activating Its Receptor [Internet].

Cell. 1996. p. 607-617. Available from: http://dx.doi.org/10.1016/s0092-8674(00)80134-8.

[21] Marqués G, Musacchio M, Shimell MJ, et al. Production of a DPP Activity Gradient in the Early Drosophila Embryo through the Opposing Actions of the SOG and TLD Proteins [Internet]. Cell. 1997. p. 417-426. Available from: http://dx.doi.org/10.1016/s00928674(00)80425-0.

[22] Wang Y-C, Ferguson EL. Spatial bistability of Dpp-receptor interactions during Drosophila dorsal-ventral patterning. Nature. 2005;434:229-234.

[23] Ashe HL, Levine M. Local inhibition and long-range enhancement of Dpp signal transduction by Sog. Nature. 1999;398:427-431.

[24] Dorfman R, Shilo BZ. Biphasic activation of the BMP pathway patterns the Drosophila embryonic dorsal region. Development. 2001;128:965-972.

[25] Umulis DM, Serpe M, O’Connor MB, et al. Robust, bistable patterning of the dorsal surface of the Drosophila embryo [Internet]. Proceedings of the National Academy of Sciences. 2006. p. 11613-11618. Available from: http://dx.doi.org/10.1073/pnas.0510398103.

[26] Wharton KA, Serpe M. Fine-tuned shuttles for bone morphogenetic proteins. Curr. Opin. Genet. Dev. 2013;23:374-384.

[27] Miles WO, Jaffray E, Campbell SG, et al. Medea SUMOylation restricts the signaling range of the Dpp morphogen in the Drosophila embryo [Internet]. Genes \& Development. 2008. p. 2578-2590. Available from: http://dx.doi.org/10.1101/gad.494808. 
[28] Kosman D. Multiplex Detection of RNA Expression in Drosophila Embryos [Internet]. Science. 2004. p. 846-846. Available from: http://dx.doi.org/10.1126/science.1099247.

[29] Carrell SN, Reeves GT. Imaging the dorsal-ventral axis of live and fixed Drosophila melanogaster embryos. Methods Mol. Biol. 2015;1189:63-78.

[30] Trisnadi N, Altinok A, Stathopoulos A, et al. Image analysis and empirical modeling of gene and protein expression. Methods. 2013;62:68-78.

[31] Shilo B-Z, Haskel-Ittah M, Ben-Zvi D, et al. Creating gradients by morphogen shuttling. Trends Genet. 2013;29:339-347.

[32] Ambrosi P, Chahda JS, Koslen HR, et al. Modeling of the dorsal gradient across species reveals interaction between embryo morphology and Toll signaling pathway during evolution. PLoS Comput. Biol. 2014;10:e1003807.

[33] Kanodia JS, Rikhy R, Kim Y, et al. Dynamics of the Dorsal morphogen gradient. Proc. Natl. Acad. Sci. U. S. A. 2009;106:21707-21712.

[34] O’Connell MD, Reeves GT. The presence of nuclear cactus in the early Drosophila embryo may extend the dynamic range of the dorsal gradient. PLoS Comput. Biol. 2015;11:e1004159.

[35] Govind S, Whalen AM, Steward R. In vivo self-association of the Drosophila rel-protein dorsal. Proc. Natl. Acad. Sci. U. S. A. 1992;89:7861-7865.

[36] Roth S, Schüpbach T. The relationship between ovarian and embryonic dorsoventral patterning in Drosophila. Development. 1994;120:2245-2257. 
[37] Roth S, Hiromi Y, Godt D, et al. cactus, a maternal gene required for proper formation of the dorsoventral morphogen gradient in Drosophila embryos. Development. 1991;112:371388.

[38] Cardoso MA, Fontenele M, Lim B, et al. A novel function for the IאB inhibitor Cactus in promoting Dorsal nuclear localization and activity in theDrosophilaembryo [Internet]. Development. 2017. p. 2907-2913. Available from: http://dx.doi.org/10.1242/dev.145557.

[39] Garcia M, Nahmad M, Reeves GT, et al. Size-dependent regulation of dorsal-ventral patterning in the early Drosophila embryo [Internet]. Developmental Biology. 2013. p. 286299. Available from: http://dx.doi.org/10.1016/j.ydbio.2013.06.020.

[40] Araujo H, Bier E. sog and dpp exert opposing maternal functions to modify toll signaling and pattern the dorsoventral axis of the Drosophila embryo. Development. 2000;127:36313644.

[41] Araujo HM, Carneiro K, Fontenele MR, et al. Delayed effect of a maternal BMP on establishment of the embryonic dorsal-ventral axis [Internet]. Developmental Biology. 2006. p. 427. Available from: http://dx.doi.org/10.1016/j.ydbio.2006.04.314.

[42] Fontenele M, Carneiro K, Agrellos R, et al. The Ca2+-dependent protease Calpain A regulates Cactus/I kappaB levels during Drosophila development in response to maternal Dpp signals. Mech. Dev. 2009;126:737-751.

[43] Fontenele M, Lim B, Oliveira D, et al. Calpain A modulates Toll responses by limited Cactus/IкB proteolysis [Internet]. Molecular Biology of the Cell. 2013. p. 2966-2980. Available from: http://dx.doi.org/10.1091/mbc.e13-02-0113. 


\section{Figures}
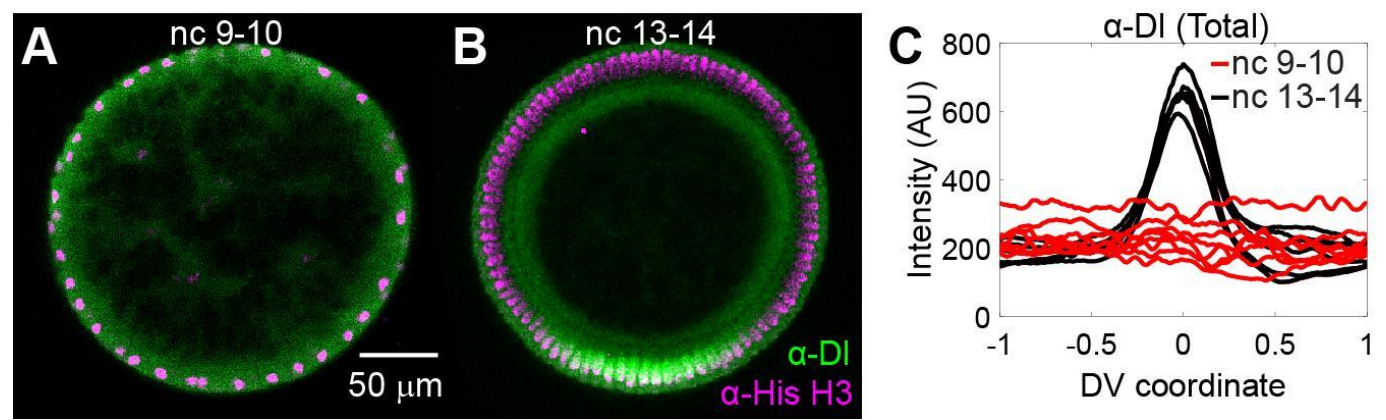

Figure 1: Accumulation of Dl on the ventral side. (A) In nuclear cycle (nc) 9-10 embryos, D1 protein (green) is distributed uniformly around the embryo. (B) In older embryos (nc 13-14), D1 protein has accumulated on the ventral side. (C) Quantification of the total Dl fluorescence intensity (nuclear + cytoplasmic) in nc 91-0 vs nc 13-14 embryos. Magenta in A and B: Histone H3 protein to mark the nucleus. Modified with permission from [17]. 

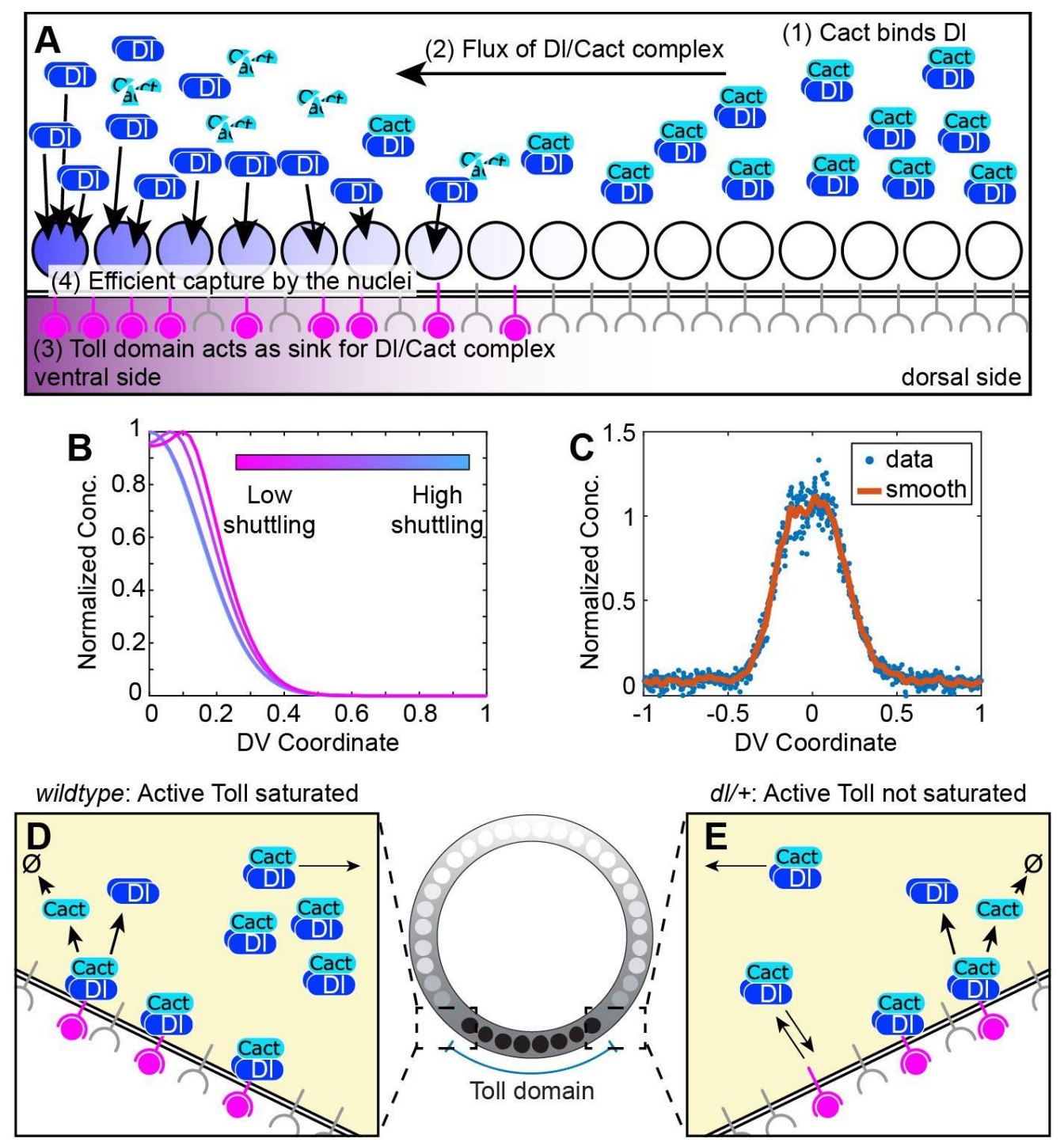

Figure 2: Description of shuttling mechanism..(A) Four biophysical processes necessary for shuttling to occur. Note that process (4), capture by the nuclei, is necessary to prevent free Dl in the cytoplasm from diffusing back to the dorsal side. (B) An example of the hallmark phenotypes that occur when the strength of shuttling is lowered: the gradient becomes wider, flatter on top, or splits into two peaks. (C) Typical embryo from mother heterozygous null for $d l$. The D1 gradient is flatter on top, and is also wider. (D) In wildtype embryos, Toll saturation likely occurs in the tail of the Toll domain. Sufficient numbers of Dl/Cact complexes saturate the active Toll signaling complex, which allows other Dl/Cact complexes to shuttle past towards the ventral 
midline. (E) In $d l /+$ embryos, there are fewer Dl/Cact complexes, so that active Toll is less saturated, and a lower fraction of D1/Cact complexes shuttle to the ventral midline.Modified with permission from [17]. 

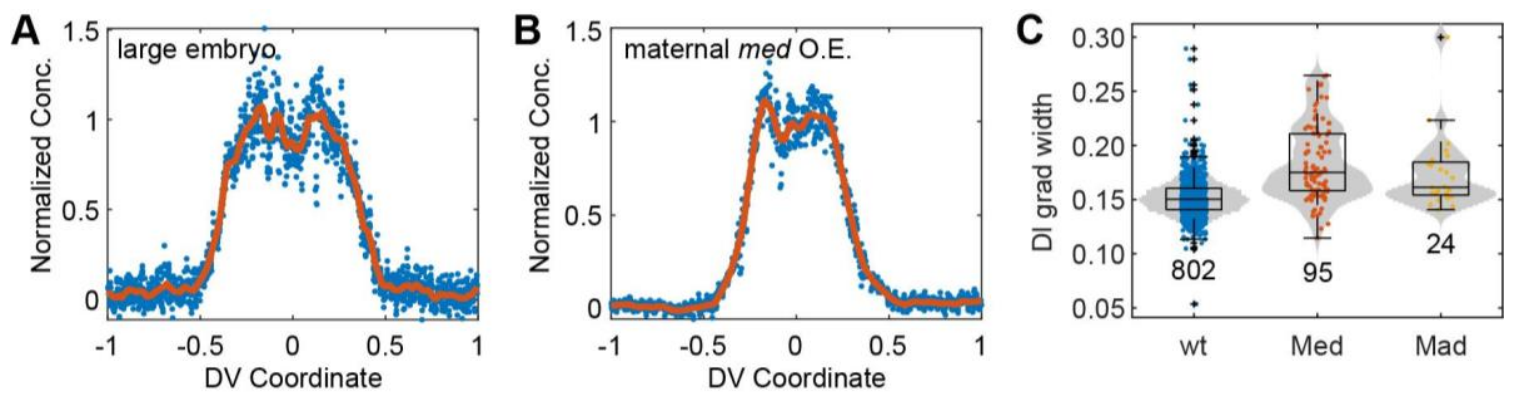

Figure 3: Shuttling phenotypes in other contexts. (A) Quantification of the D1 gradient in embryos with very large DV axes reveals a split-peaked embryo. It is possible that shuttling is responsible for size-dependent scaling of the Dl gradient, which breaks down when the DV axis becomes too large. Modified from [39]. (B) The hallmark phenotypes of shuttling also appear in embryos that have maternally overexpressed components of the BMP pathway. (C) Box-andviolin plot of measure of the Dl gradient width in wildtype, Med-GFP O.E., and Mad-GFP O.E. embryos. Both the Med- and Mad-GFP O.E. embryos have statistically wider gradients (p-val < $\left.10^{-150}\right)$. 